\title{
Safety and the pandemic: changing perspectives for patients, professionals and populations
}

\author{
DOI: $10.7861 /$ fhj.ed.8.3.3
}

Safety is central to patient care but our understanding of how we ensure and manage safe care is changing. This edition of the Future Healthcare Journal brings together reviews and opinion pieces from some leading thinkers and practitioners in the field, as well as some practical examples of safety improvement. While most of this work and thinking was developed before the COVID-19 pandemic, we believe that the perspectives of patients and families, professionals, and populations on healthcare safety have been significantly changed by the recent experience. Fortunately, these changing perspectives align with our developing understanding of healthcare safety and how we should work to maximise care outcomes and prevent harm.

Firstly, the concepts of hazards and risks are understood in practice, with COVID-19 infection being a hazard for illness or even death, with multiple factors that might increase or reduce your risk as an individual or family in contracting the disease or having a poor outcome. Gathering more information about the risk helps people to then make choices based on their level of understanding, their risk tolerance and the acceptability of any barriers. Some barriers are simpler to implement widely (eg vaccination), while others (eg social distancing) are more difficult. We also developed a greater understanding of levels of risk, the changing prevalence of the hazard and the effectiveness of barriers. To protect people, we rapidly redesigned the use of physical spaces, reduced travel and increased ventilation, and tried to understand the impact of these changes on the way we work. New ways of working were assessed against metrics of risk and were scaled at a speed previously unheard of, locally, nationally and internationally. What worked was passed on and spread: a large-scale application of the concept of Safety II, collective learning from contextualised practice, and copying systems that deliver under pressure and that can be applied to many patients and practitioners. ${ }^{1}$ The experience of using assessments of risk and harm to drive change at pace and scale will be one of the lasting legacies of COVID-19.

More than anything, improvements in safety depend on the development of robust systems to deliver reliable processes that result in improved safe care outcomes: our response to the pandemic and the learning from what has worked and what went wrong has also developed our understanding of the importance of systems, their interdependence and important components. So, when oxygen supplies became low, we began to understand that the weather, the supply, the geography and guidance for clinical use were affecting this, and we connected with others who had learned how to respond, even involving non-medical industries to support.

Weaver and colleagues give us a helpful review of some of the key aspects of patient safety, and how the Health Services Investigation Branch has learned from other industries' systems approaches to safety. ${ }^{2}$ They share how the Systems Engineering
Initiative for Patient Safety (SEIPS) investigation model explores system factors that may have resulted in an adverse event. This will be rolled out across the NHS as part of the Patient Safety Incident Response Framework approach and the Patient Safety Syllabus. ${ }^{3,4}$ One other theme of the Patient Safety Syllabus is 'human factors'. This is a commonly quoted term in healthcare safety, but understanding it is, arguably, limited in healthcare staff. Sujan and colleagues' review of ergonomics and human factors gives us a greater understanding of the importance of human factors for the delivery of health services. ${ }^{5}$ They also point us towards opportunities to grow our understanding more and use it in designing safer healthcare.

Given that care is increasingly delivered in complex multidisciplinary teams, the papers from Guest et al, and Chenevert and Bascombe are timely reminders of the importance of curriculum design for new members of the team and the impact of physician associates on the safety of care.,7

This is a global pandemic and our learning for healthcare safety should be global, as should be our response. We must therefore ensure that our learning for healthcare safety is shared and spread. The reach of the Institute of Global Health Innovation and the International Society for Quality in Health Care (ISQua) across nations is collectively unique: O'Brien et al collate global safety policy, case studies and recommendations that should be applied post-pandemic. ${ }^{8}$ These include how Sierra Leonne learned for the COVID-19 pandemic from Ebola, this learning needs to be shared more widely.

Greater use of digital technologies will be a lasting hallmark from the pandemic and, as with many interventions, videoconferencing (as one example) was introduced at speed without full understanding of risks and benefits. While undoubtedly the risks of travelling to hospital were too high in the early phases, the balance of the risk/benefit ratio of remote consultation is less clear now that we have better understanding of managing infection risks. Flott and colleagues remind us of the many facets of safety in the usage of digital technologies, technologies that are rapidly becoming a central part of our safety systems. ${ }^{9}$

Perhaps the biggest shift both in the pandemic and for the future is patients and the public taking a greater ownership and leadership of healthcare safety. Four papers in this edition demonstrate what might be possible if we open our minds and our cultures to see patients and their families as true partners in safer healthcare, more accurate records, earlier recognition of deterioration and better safety checks, just to start with; key safety priorities for us all. ${ }^{10-13}$

And finally, understanding hazards, risks and barriers is of little more than intellectual benefit if we can't deliver locally contextualised improvement: Flatman's practical guide to medication safety at transfer from hospital will help teams to get on with improvement projects. ${ }^{14}$ The paper also shows great learning on how to develop guidance with, once more, the prominent role of patients as their own champions and guardians of safe use of medications. 
John Dean

Clinical director for quality improvement and patient safety, Royal College of Physicians, London, UK Consultant physician, Ysbyty Gwynedd, Bangor, UK, senior clinical lecturer, Bangor University, Bangor, UK and improvement science fellow, The Health Foundation, London, UK

\section{References}

1 Hollnagel E, Leonhardt J, Licu T, Shorrock S. From Safety-I to Safety-II: A White Paper. EUROCONTROL, 2013.

2 Weaver S, Stewart K, Kay L. Systems-based investigation of patient safety incidents. FH] 2021;8:e593-97.

3 NHS England, NHS Improvement. Patient Safety Incident Response Framework. NHS, 2020. www.england.nhs.uk/patientsafety/incident-response-framework [Accessed 22 October 2021]

4 Health Education England. Patient Safety Syllabus. HEE, 2021. www. hee.nhs.uk/our-work/patient-safety [Accessed 22 October 2021].

5 Sujan M, Pickup L, Bowie P et al. The contribution of human factors and ergonomics to the design and delivery of safe future healthcare. FHJ 2021;8:e574-79.
6 Guest BN, Chandrakanthan C, Bascombe K, Watkins ]. Pharmacology for physician associate programmes: a collaborative, flexible and responsive approach to curriculum design. FHJ 2021;8:e580-84.

7 Chenevert L, Bascombe K. Physician associates advance patient safety. FHJ 2021;8:e613-15.

8 O'Brien N, Durkin M, Lachman P. Lessons post-COVID-19 from national and international approaches to safety and quality in healthcare. FHJ 2021;8:e602-8.

9 Flott K, Maguire J, Phillips N. Digital safety: the next frontier for patient safety. FHJ 2021;8:e598-601.

10 Subbe CP, Ahsan S, Smith L, Frey Renggli J. When patients (and families) raise the alarm: An audible patient voice: How can we ensure that patients are treated as partners in their own safety? FH] 2021;8:e564-66.

11 Lam BD, Bourgeois F, DesRoches CM, Dong Z, Bell SK. Attitudes, experiences, and safety behaviours of adolescents and young adults who read visit notes: Opportunities to engage patients early in their care. FHJ 2021:8:e585-92.

12 Bucknall T, Quinney R, Booth $L$ et al. When patients (and families) raise the alarm: Patient and family activated rapid response as a safety strategy for hospitals. FHJ 2021;8:e609-12.

13 Harris K, Russ S. Patient-completed safety checklists as an empowerment tool for patient involvement in patient safety: concepts, considerations and recommendations. FH] 2021;8:e567-73.

14 Flatman J. How to improve medication safety at hospital discharge: let's get practical. FH] 2021;8:e616-18. 